\title{
Cooperative Spectrum Sensing and Determination of Optimum Threshold in Cognitive Radio System
}

\author{
M.M. Abdullahi \\ Department of Electrical and \\ Computer Engineering \\ Ahmadu Bello University, \\ Zaria.
}

\author{
S.M. Sani \\ Department of Electrical and \\ Computer Engineering \\ Ahmadu Bello University, \\ Zaria.
}

\author{
M.B. Mu'azu \\ Department of Electrical and \\ Computer Engineering \\ Ahmadu Bello University, \\ Zaria.
}

\begin{abstract}
The challenges associated with the determination of detection threshold due to uncertainty was addressed in this paper. The technique of Cooperative Sensing Spectrum (CSS) was used to model the functional subsystems that made up the Cognitive Radio System. The model developed utilizes channel centre frequencies. Also, implemented and threshold actualized for the presence/absence of PU based on false alarm and detection decisions. The innovative approach employed the use of FLA for the improvement of the detection threshold. This was used to fine tune and determine the optimum threshold using the hypothesis $\mathrm{H}_{0}$ and $\mathrm{H}_{1}$.
\end{abstract}

\section{Keywords}

CSS, FLA, PU and SU

\section{INTRODUCTION}

Cognitive radio, an emerging intelligent wireless access and communication scheme that is aware of its surrounding environment to allow changes in certain operating parameters for the objective of providing reliable communications and efficient utilization of the radio spectrum [1]. It is based on the concept of dynamic spectrum access, whereby spectrum licensed holders, the Primary Users (PU) grant permission for spectrum access to non-licensed Secondary Users (SU). As long as PU maintain a reliable Quality of Service (QoS) for its own operations, as well as interference to PU activity is minimal and confined [2][3]. To achieve this goal, SU must actively sense PU spectrum (Spectrum Sensing) to detect spectrum holes/opportunity. Spectrum opportunity involves utilizing currently not in use spectrum by the PU. While, Spectrum Sensing (SS) involves spectrum of interest sensing which can be utilized by the SU without causing interference to the PU [4][1][5].

Spectrum Sensing (SS) poses new challenges in both hardware and software. This could be in the radio front end to operate over wide frequency ranges, high speed signal processors, efficient system, or algorithms to process computation demands etc [6]. PU activity changes from time to time, hence SS has to be performed in a periodic manner, thus, forming a sensing cycle [7] SS results in one of two decisions; false alarm and detection. In false alarm, the SS declares PU is present when the spectrum is empty. In detection, the SU correctly declares a PU is using the spectrum [8]. The performance of sensing detection is thus measured through the probability of these two events. These two metrics however, are conflicting parameters and the ideal case of perfect detection performance is rarely achievable. Therefore in practice, the PU and SU specifies a set of detection requirements based on the maximum interference the PU can tolerate and the minimum spectral efficiency required in operating the SU network [9][10] specifies that "Spectrum decision is best performed by combining spectrum analysis results from all nodes within the SU network".

SS techniques are classified into either non-cooperative or cooperative. In this paper cooperative sensing spectrum (CSS) is used because of its ability to performs
i. Own local spectrum sensing measurement independently; and

ii. Binary decision using the "OR" logic.

\section{COOPERATIVE SPECTRUM SENSING (CSS) SYSTEM}

The primary idea of CSS is to enhance the spectrum sensing performance by exploiting the spatial diversity in the observations of spatially located SU. Since it is unlikely that all spatially distributed SU in a cognitive radio environment will concurrently experience the fading or receiver uncertainty problem. Hence, when users collaborate and share the SS results among themselves, the combined cooperative decision derived from the spatially collected observations can overcome the deficiency of individual observation of each SU.

\section{THRESHOLD DETECTION IN COGNITIVE RADIO}

The need for precise threshold selection in the CSS solution comes to the fore considering the Cognitive Radio Network's inherent drawbacks (factors) of noisy channel, fading effect of signal strength and different radio operating environment. These factors make a single threshold value unsuitable for sensing in a Cooperative Cognitive Radio model employing Energy Detector as the sensing technique. To counteract this drawback, threshold determination is performed from knowledge of the received signal energies and their probability distribution in real time, while the cognitive radio is operational. Matinmikko 2008 established that very low Signal to Noise Ration (SNR) diminishes the suitability of choosing an Energy Detector for the sensing part of a Cognitive Radio couple with uncertainty in sensing the PU [11]. Differential mathematical representations of this uncertainty exist and are given as [12]:

$$
\begin{aligned}
& P_{f a}=\frac{1}{2} \operatorname{erfc}\left(\frac{V_{-} t-2 T W}{2 \operatorname{sqrt}(2) \operatorname{sqrt}(T W)}\right) \\
& P_{D}=\frac{1}{2} \operatorname{erfc}\left(\frac{\left(V_{t}-2 T W\right)-\lambda}{2 \operatorname{sqrt}(2) \operatorname{sqrt}(T W)+\lambda}\right)
\end{aligned}
$$

The probability of missed detection is 


$$
\begin{gathered}
P_{m d}=1-P_{\mathrm{D}} \\
\operatorname{erfc}(\mathrm{z})=\frac{2}{\pi} \int_{z}^{\infty} \exp \left(-x^{2}\right) d x
\end{gathered}
$$

The non-central chi-square distribution is:

$$
\lambda=\frac{1}{N_{o}} \int_{0}^{T} s^{2}(t) d t=\frac{E_{s}}{N_{o}}
$$

Where,

$\mathrm{T}$ is the period based on time interval

$\mathrm{V}_{\mathrm{T}}$ is threshold

$\mathrm{W}$ is bandwidth of pre-filter

$\mathrm{E}_{\mathrm{s}}$ is signal energy value during time $\mathrm{T}$

$\mathrm{N}_{\mathrm{o}}$ is noise power spectrum density (two sided)

For simulation purposes in Matlab environment, the MarcumQ function is used for the computation of the probabilities. The Probability Distribution is represented using the MarcumQ function as:

$$
\mathbf{Q}=\operatorname{marcumq}(\mathbf{a}, \mathbf{b}, \mathbf{m})
$$

Which computes the generalized Marcum Q, defined by

$$
Q(a, b)=\frac{1}{a^{a-1}} \int_{b}^{\infty} x^{m} \exp \left(-\frac{x^{2}+a^{2}}{2}\right) I_{m-1}(a x) d x
$$

Where,

$\mathrm{a}$ and $\mathrm{b}$ are nonnegative real numbers,

$\mathrm{m}$ is a positive integer,

$I_{m-1}$ is the modified Bessel function of the first kind of order $\mathrm{m}-1$.

When any of the inputs is a scalar, it is expanded to the size of the other inputs. For the $\mathrm{P}_{\mathrm{fa}}$, the gamma function used is given by:

$$
P(a, x)=\frac{1}{\Gamma(a)} e^{-t} t^{a-1} d t
$$

For any $\mathrm{A} \geq 0$, Gammainc $(\mathrm{X}, \mathrm{A}) \rightarrow 1$ as $\mathrm{X} \rightarrow \infty$.

For small $\mathrm{X}$ and $\mathrm{A}$, gammainc $(\mathrm{X}, \mathrm{A}) \approx \mathrm{X}^{\mathrm{A}}$, and gammainc $(0,0)=1$.

The transmission characteristics of the channel is the relationship between the energy of the transmitted signal at the various probabilities computed as a probability distribution function (PDF). Figure 1 described chi-squared distribution for energy thresholds.

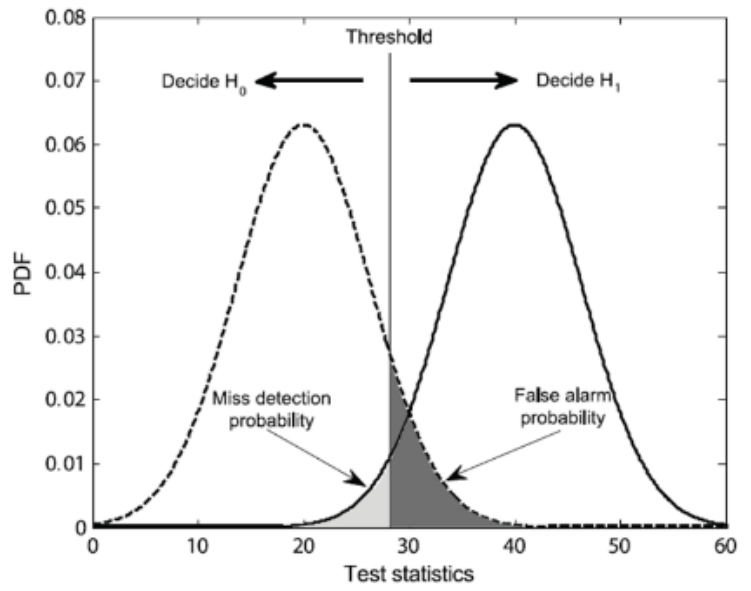

Figure 1: Probability vs. Energy Plot using Chi-square Distribution [13]

The Chi-square distribution relates the probabilities to signal energy for the case when PU is;

i. Absent and only noise energy is being sensed $\left(\mathrm{H}_{0}\right.$ hypothesis) and

ii. Present and transmission is of both the noise and signal energy ( $\mathrm{H}_{1}$ hypothesis)

These two cases are clearly shown Figure 3

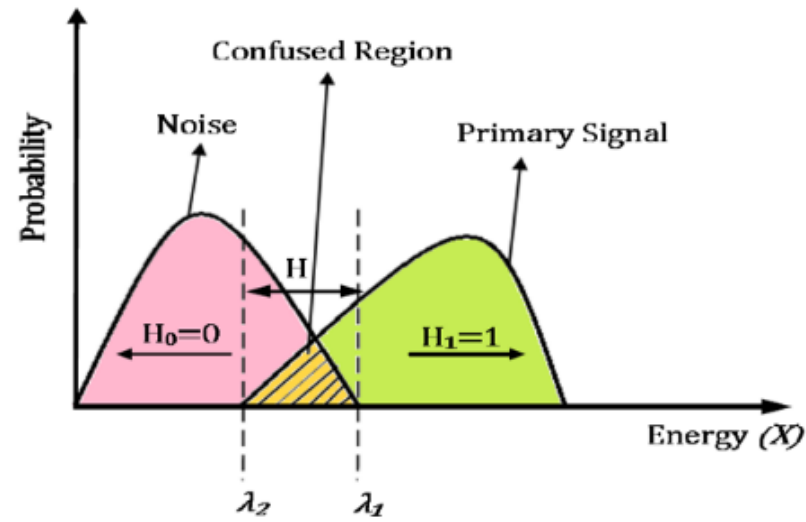

Figure 2: Probability vs. Energy Plot Showing Chi-square Distribution [13]

From Figure 2, the region of intersection of the two curves (between $\lambda_{1}$ and $\lambda_{2}$ ) is the uncertainty region. This uncertainty region is the region in which the Cognitive Radio has the highest probability of either missing a detection opportunity because the threshold was set too high (detection) or too low (false alarm). These uncertainty results in simultaneous transmission of PU and SU.

\section{METHODOLOGY}

The complete MATLAB Simulink model of a Cognitive Radio System (CRS) is shown in Figure 3 consisting of various functional subsystems. 


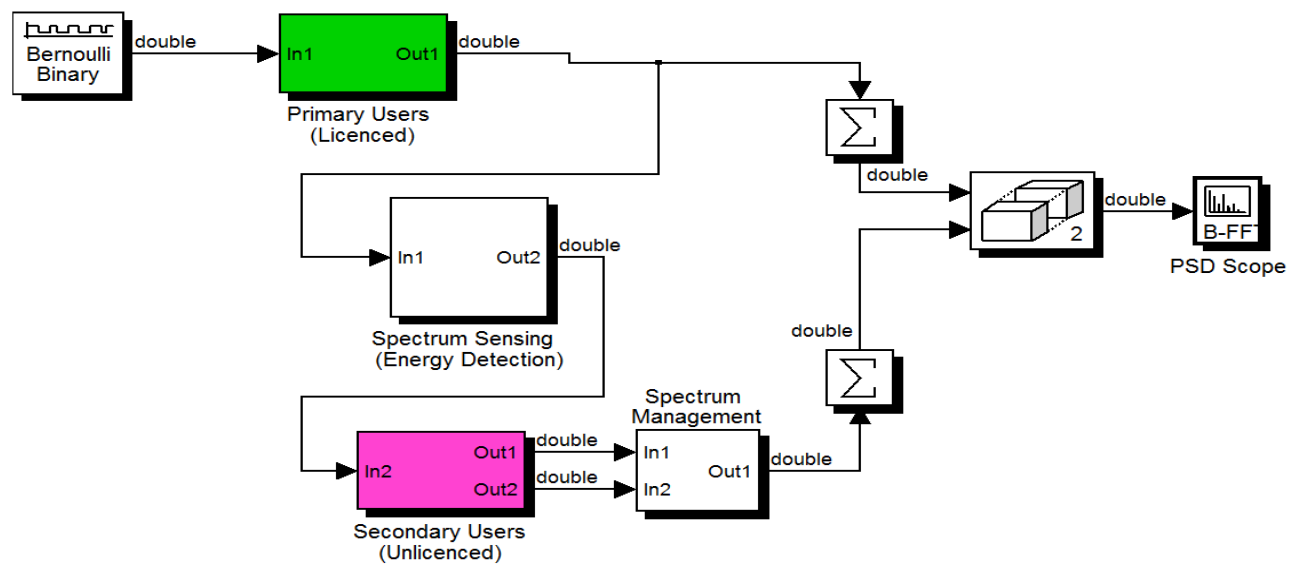

Figure 3: MATLAB Simulink of the CRS model.

The functional subsystems that made up the Cognitive Radio System of Figure 3 are; PU Generator, Transmission channel for signal propagation, SS subsystem, SU Generator, and Allocation or Spectrum Management (SM) subsystem. The methods used involves:

i. Designing the functional subsystems of the CRS;

ii. Threshold detection selections using CSS; iii. Development of the Fuzzy Logic Algorithm (FLA) for (ii); and

iv. Simulation results and discussions

\subsection{Designing the functional subsystems of the CRS}

The developed MATLAB/Simulink of the functional subsystems of the CRS are shown in Figures $4-9$.

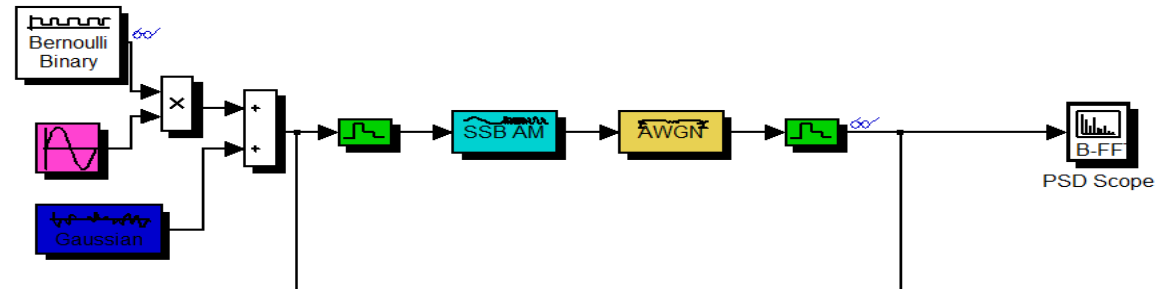

Figure 4: PU Subsystem with signal generator

The role of the designed PU in Figure 4 serves to mimic the opportunistic or random access characteristics of the PU behaviour when making use of the spectrum resource or transmitting over the channel.

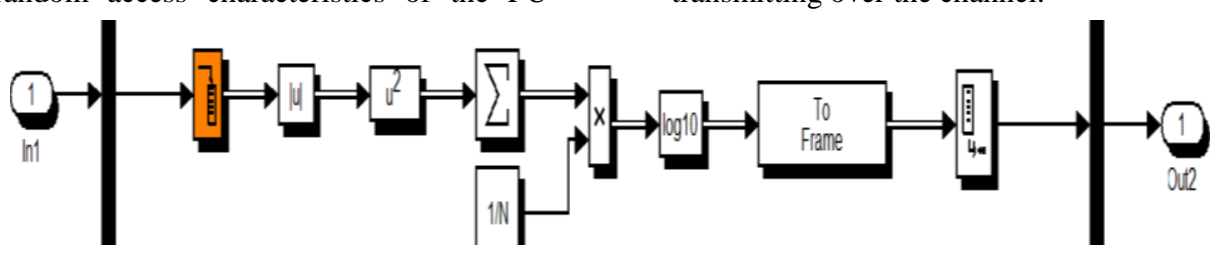

Figure 5: Energy detection based SS subsystem

The transmission channel represents the medium of signal propagation of both the PU and SU signals. In the MATLAB/Simulink model of the CRS of Figure 5, an Additive White Gaussian Noise (AWGN) block is used to describe and model the disturbances, noise, and interference sources to be found on the transmission channel. Also, Energy detection was used as the SS techniques due to its susceptibility to noise in threshold determination.

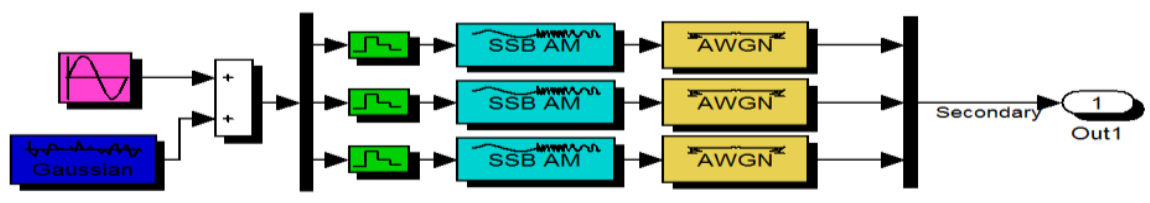

Figure 6: SU Subsystem with 3 SU. 
Figure 6 shows the SU subsystem with 3 SU. The 3 SU are equipped with sensitive front end part for detecting transmission activities on the channel. This is achieved by setting detection threshold for the PU signal using $\mathrm{H}_{0}$ and $\mathrm{H}_{1}$. Using an efficient Fuzzy Logic algorithm (FLA) for interference and decision making in the CRS so as to make accurate measurements of the signal energy on the spectrum and also carry out timely allocation and de-allocation tasks for each SU.

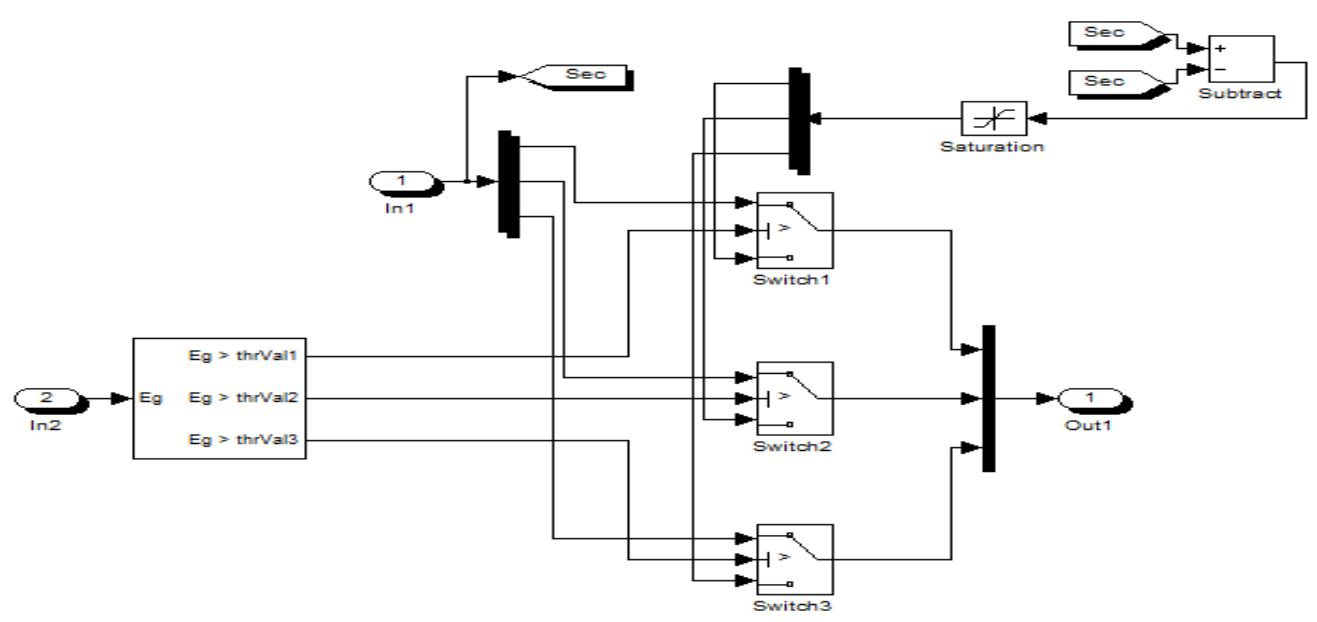

Figure 7: Spectrum Management Subsystem

This subsystem is responsible for the coordination of spectrum assignment to the $\mathrm{SU}$ and also allowing for de-allocation whenever the PU is back to transmit as the original user of the spectrum. It aids in visualization of the signal behaviours of the PU and $\mathrm{SU}$ as the common receiver is operational. It serves to implement the logic for turning ON and OFF of the SU depending on the functional behaviour at any time. This is coordinated by the fusion of the FLA.

\subsection{Threshold detection selections using CSS}

Figure 8 shows the working flow chart of the CSS system with the algorithm (FLA) blocks for it development.

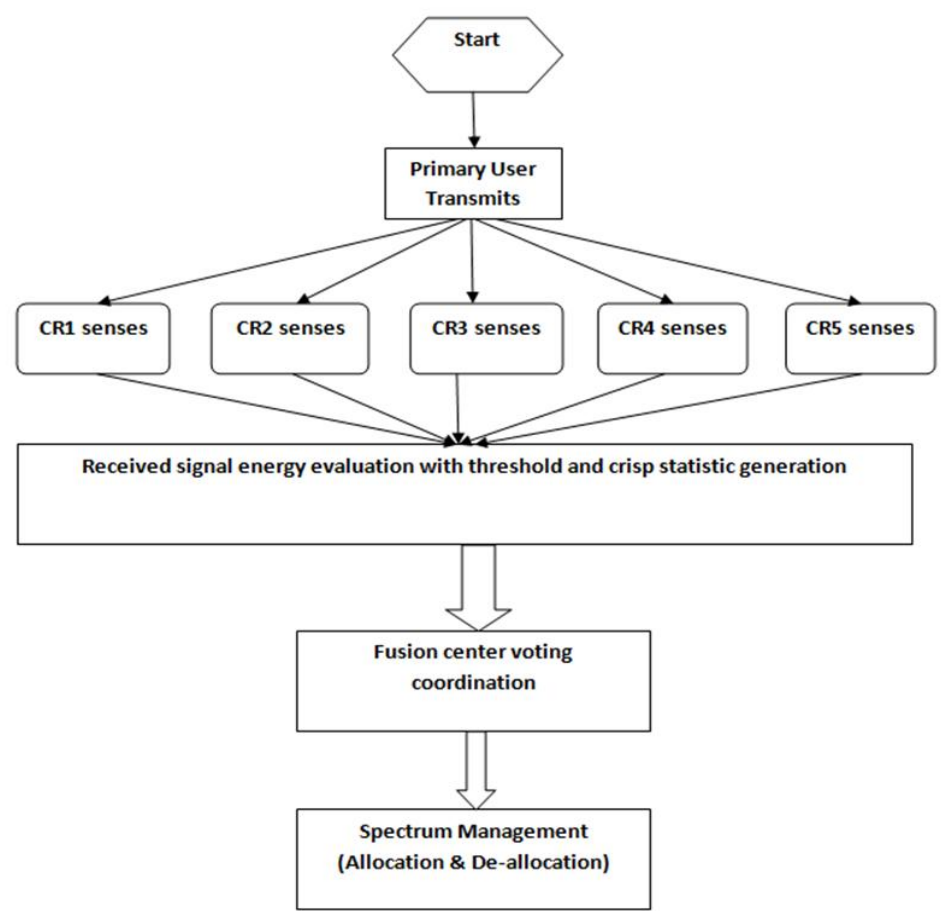

Figure 8: Flowchart for Threshold detection based on CSS 
The fusion centre in Figure 8 plays the role of collecting all the individual cognitive radio results and counting the votes based on presence or absence of PU. It is the most important aspect of the CSS Cognitive radio model, as this functionality is what handles the cooperation in the cooperative cognitive radio.

\subsection{Development of the Fuzzy Logic Algorithms (FLA)}

The FLA used in simulating the developed Cooperative Spectrum Sensing was developed based on the flow chart in Figure 9

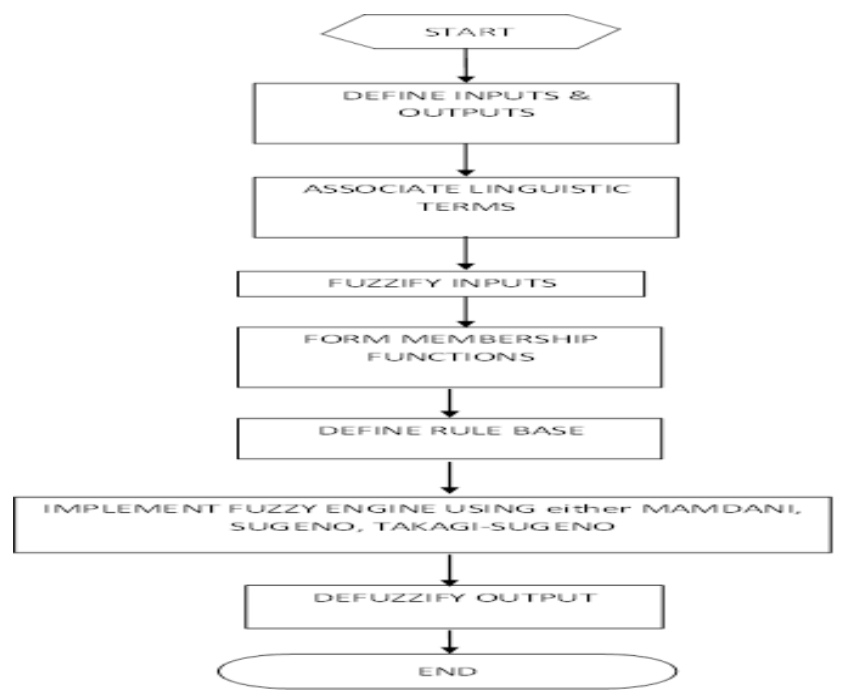

Figure 9: The Fuzzy Logic Algorithm

The description of each of the stages of the flow chart of Figure 9 thus are:

A. Fuzzy Variables: The variables used in the Fuzzy Decision Engine (FDE) are:

i. Inputs: SNR and Energy; and

ii. Output: PDF.

iii. Universe of Discourse for the variables chosen have the following ranges (carefully chosen from prior knowledge of the value space taken by these variables):
a. SNR:
$10-15 \mathrm{~dB}$,
b. Energy:
$0-130 \mathrm{~dB} / \mathrm{W}$
c. Probabilities:
$0-1$.

B. Linguistic Terms: These variable values are mapped to linguistic terms to describe their degree of belonging within the ranges given. The linguistic terms used are called fuzzy sets and in this work takes the form Low (L) or Very Low (VL), Medium (M), High (H) or Very High (VH).

C. Fuzzy Membership functions: From (B), suitable simple Fuzzy Membership Functions (FMF) (triangular and trapezoidal) were used to describe the fuzzy sets. Subsequently, the FMF are used to fine tune the (FDE) to obtain a precise representative output.

D. Fuzzy Rule Table: Fuzzy rule Table is generated from the input variables as shown in Table 1.
Table 1: Fuzzy Rule Table with 3 Linguistic Terms for Both Inputs

\begin{tabular}{|c|c|c|c|}
\hline Energy SNR & Ls & Ms & Hs \\
\hline Le & L & L & M \\
\hline Me & L & M & H \\
\hline He & M & H & H \\
\hline
\end{tabular}

E. Rule Base: Based on Table 1, an initial fuzzy rule base is formed as follows:

$$
\begin{aligned}
& \text { If }(\mathrm{SNR}=\mathrm{Ls} \text { and energy=Le) then } \mathrm{PDF}=\mathrm{L} \\
& \text { If }(\mathrm{SNR}=\mathrm{Ls} \text { and energy=Me) then } \mathrm{PDF}=\mathrm{L} \\
& \text { If }(\mathrm{SNR}=\mathrm{Ls} \text { and energy=He) then } \mathrm{PDF}=\mathrm{M} \\
& \text { If }(\mathrm{SNR}=\mathrm{Ms} \text { and energy=Le) then } \mathrm{PDF}=\mathrm{L} \\
& \text { If }(\mathrm{SNR}=\mathrm{Ms} \text { and energy=Me) then } \mathrm{PDF}=\mathrm{M} \\
& \text { If }(\mathrm{SNR}=\mathrm{Ms} \text { and energy=He) then } \mathrm{PDF}=\mathrm{H} \\
& \text { If }(\mathrm{SNR}=\mathrm{Hs} \text { and energy=Le) then } \mathrm{PDF}=\mathrm{M} \\
& \text { If }(\mathrm{SNR}=\mathrm{Hs} \text { and energy=Me) then } \mathrm{PDF}=\mathrm{H} \\
& \text { If }(\mathrm{SNR}=\mathrm{Hs} \text { and energy=He) then } \mathrm{PDF}=\mathrm{H}
\end{aligned}
$$

The initial Fuzzy Rule Base are implemented in the MATLAB'S Fuzzy Toolbox and to obtain the PDF output. Visualization of the Fuzzy Logic implementation is done by making a plot of PDF against energy 


\subsection{Simulation Results and Discussions}

The modulated PU and SU signal generations are thus shown in Figures 10 and 11.

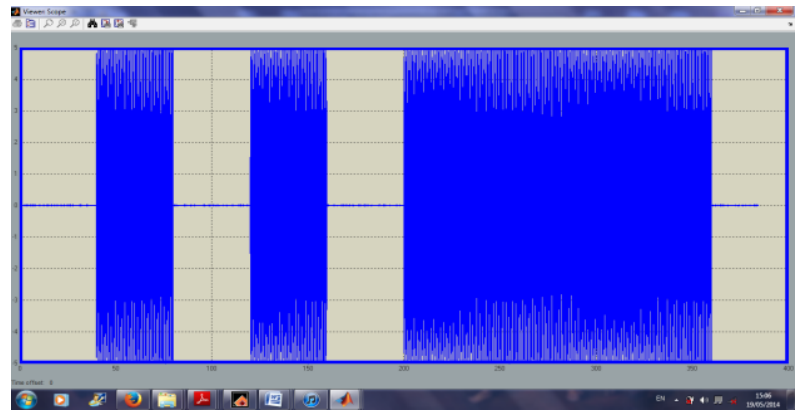

Figure 10: Stochastic Primary User Behaviour

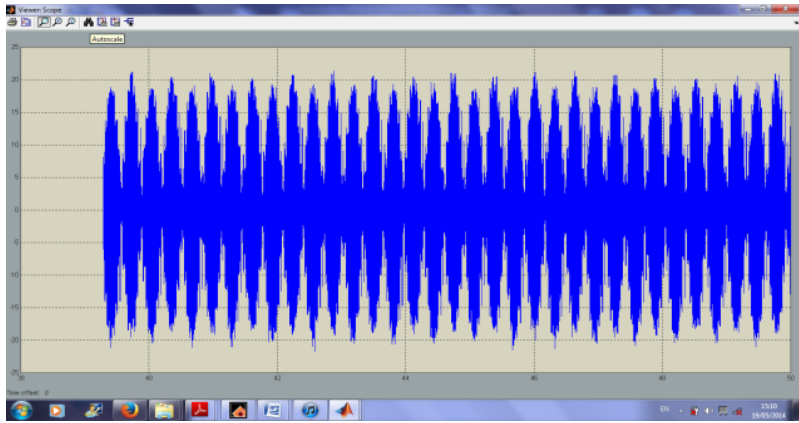

Figure 11: Modulated Secondary User Signal

Figures 10 and 11 show that the modulated PU and SU signals are stochastic and continuous in nature respectively. The Bernoulli random number generator compares the sensed energy against the threshold to determine presence or absence of PU. The energy detector makes use of the sum and average of the energy signal in order to calculate the covariance of the energy. Thereby generating the two (2) hypothesis; $\mathrm{H}_{0}$ and $\mathrm{H}_{1}$. when the $\mathrm{PU}$ signal is $\mathrm{ON}$ indicating the Bernoulli probability of zero parameter is set to a value not equal to 1 and OFF when the Bernoulli probability of zero parameter is set to 1 or absolute certainty. Figure 12 shows different scenarios of allocation or Spectrum Management.

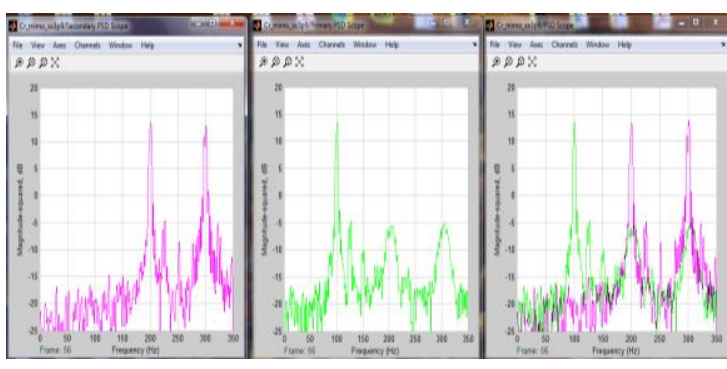

(a)

(b)

(c)

Figure 12: Scenarios of Allocation or Spectrum Management

From Figure 12, the model developed has 3 each of PU, SU and transmission channels. Also, each with centre frequency of $100 \mathrm{~Hz}, 200 \mathrm{~Hz}$ and $300 \mathrm{~Hz}$. The PU were transmitted using on channel centre frequency. Also simulated at 100, 200 and $300 \mathrm{~Hz}$; The PU plot is the middle plot b (Green) while the SU occupies the leftmost plot a (purple), while the extreme right being the combined PU/SU spectrum management (allocation/de-allocation) plot showing the behaviour of the Cognitive Radio spectrum management subsystem by highlighting the allocation and de-allocation process of a SU on the Cognitive Radio network. From the results of Figure 12, when the PU is actively transmitting on the centre frequency of $100 \mathrm{~Hz}$, the $\mathrm{SU}$ occupies the other free bands at 200 and $300 \mathrm{~Hz}$ respectively.

Simulations were carried out for different possible spectrum allocations between the PU and SU. The simulations results shown in Figures 13, 14, 15 and 16.

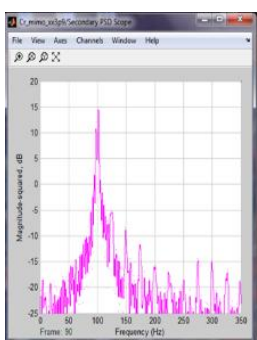

(a)

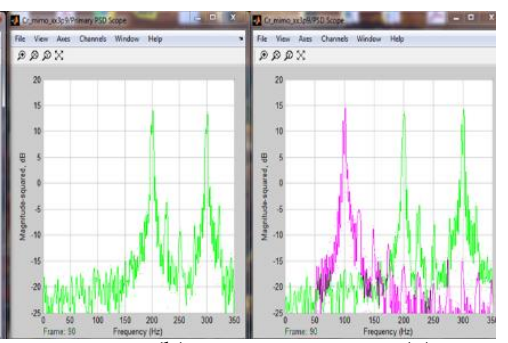

(b) (c)
Figure 13: spectrum allocation with PUs 2 and 3 active

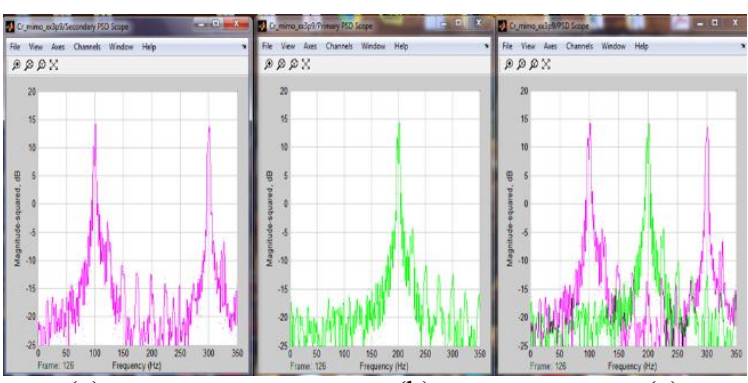

(a)

(b)

(c)

Figure 14: Spectrum allocation with primary user 2

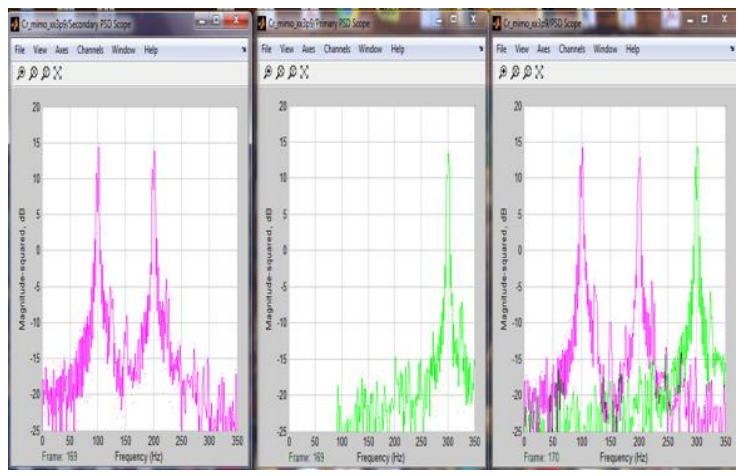

(a)

(b)

(c)

Figure 15: Spectrum allocation with primary user 3 active

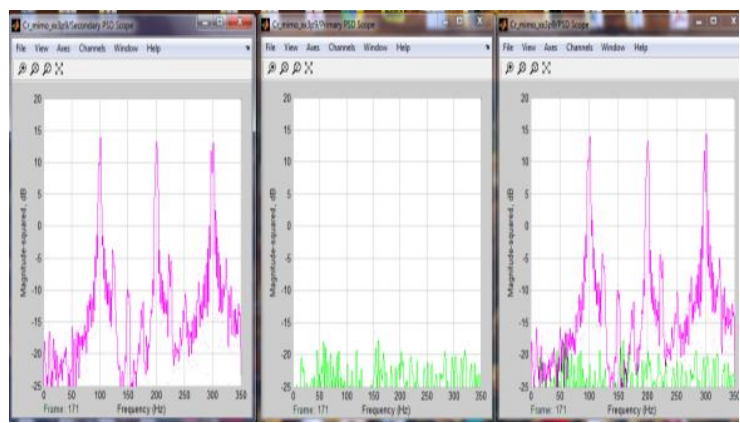

(a)

(b)

(c)

Figure 16: Spectrum allocation with no active primary user 
Figure $13 \mathrm{~b}$ shows the spectrum allocation with PU 2 and 3 active. Allocating the free spectrum band requires that only SU 1 (Figure 13a) can transmit simultaneously. This is clearly depicted in Figure 13c. The pattern is similar to Figures $14-$ 16. It can be deducted that the CSS based model successful identify available unused bands of PU with the capability of effective usage by SU.

Visualization of the FLA implementation as to detection capability was ascertained by plotting PDF vs Energy as well with SNR. This is shown in Figures 17 and 18.

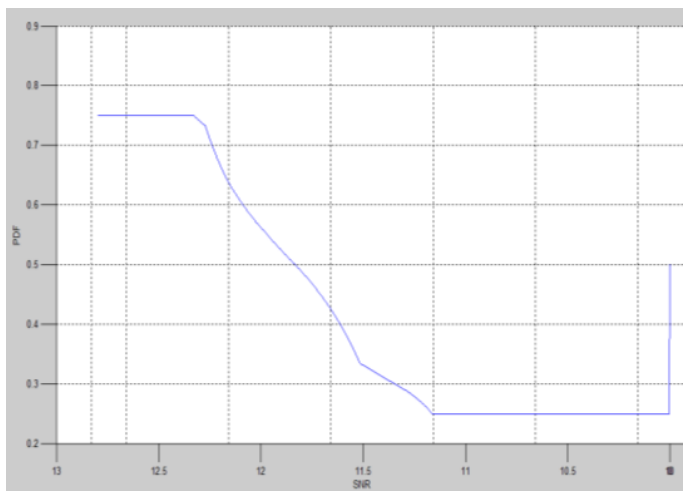

Figure 17: Plot of PDF vs SNR

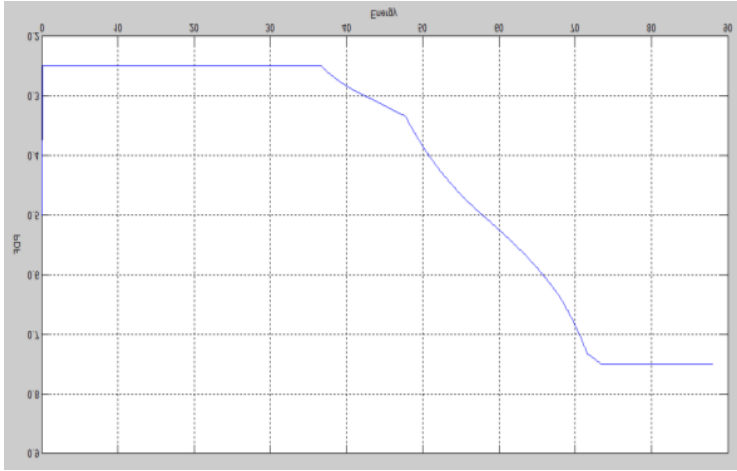

Figure 18: Energy vs. PDF

Inference from Figures 17 and 18 shows enhanced detection is prominent with reduced and increased SNR and Energy respectively.

\section{CONCLUSIONS}

The technique of CSS was used to model the functional subsystems that made up the Cognitive Radio System that is; PU Generator, Transmission channel for signal propagation, SS subsystem, SU Generator, and Allocation or Spectrum Management (SM) subsystem. The model developed utilizes channel centre frequencies. Also, implemented and threshold actualized for the presence/absence of PU based on false alarm and detection decisions. The innovative approach employed the use of FLA for the improvement of the detection threshold. This was used to fine tune and determine the optimum threshold using the hypothesis $\mathrm{H}_{0}$ and $\mathrm{H}_{1}$. Inference from the results shows improved detection is prominent with reduced and increased SNR and Energy respectively.

\section{REFERENCES}

[1] Haykin, Simon. "Cognitive radio: brain-empowered wireless communications." Selected Areas in Communications, IEEE Journal on 23.2 (2005): 201-220.

[2] Hossain, Ekram, and Vijay K. Bhargava, eds. Cognitive wireless communication networks. Springer Science \& Business Media, 2007.

[3] Mitola III, Joseph, and Gerald Q. Maguire Jr. "Cognitive radio: making software radios more personal." Personal Communications, IEEE 6.4 (1999): 13-18.

[4] Horne, W. D. "An adaptive Spectrum Access: Using the full spectrum space, technical report, the MITRE corporation (2004).

[5] Liang, Ying-Chang, et al. "Sensing-throughput tradeoff for cognitive radio networks." Wireless Communications, IEEE Transactions on 7.4 (2008): 1326-1337.

[6] Song, Yang, Yuguang Fang, and Yanchao Zhang. "Stochastic channel selection in cognitive radio networks." Global Telecommunications Conference, 2007. GLOBECOM'07. IEEE. IEEE, 2007.

[7] Ghasemi, Amir, and Elvino S. Sousa. "Optimization of spectrum sensing for opportunistic spectrum access in cognitive radio networks." 2007 4th IEEE Consumer Communications and Networking Conference. 2007.

[8] Cabric, Danijela, Artem Tkachenko, and Robert W. Brodersen. "Experimental study of spectrum sensing based on energy detection and network cooperation." Proceedings of the first international workshop on Technology and policy for accessing spectrum. ACM, 2006.

[9] Cavalcanti, Dave, et al. "Cognitive radio based wireless sensor networks." Computer Communications and Networks, 2008. ICCCN'08. Proceedings of 17th International Conference on. IEEE, 2008.

[10] Zhang, Tao, Bin Wang, and Zhiqiang Wu. "Spectrum assignment in infrastructure based cognitive radio networks." Aerospace \& Electronics Conference (NAECON), Proceedings of the IEEE 2009 National. IEEE, 2009.

[11] Matinmikko, Marja, et al. "Cognitive radio: An intelligent wireless communication system." VTT Technical Research Centre of Finland, Tech. Rep (2008).

[12] Hao, Junwei, and Yunxiao Zu. "An enhanced energy detection algorithm in cognitive radio." Wireless Communications, Networking and Mobile Computing, 2009. WiCom'09. 5th International Conference on. IEEE, 2009.

[13] WANG, Xiaoxiang, et al. "Cooperative Spectrum Sensing Exploiting a Certain Number of CR Users Based on Multi-Antennas in Cognitive Radio Networks." China Communications 7.4 (2010): 149-155. 